\title{
El Holocausto en el aula de Secundaria: confluencia de historia, ficción y realidad en la literatura concentracionaria
}

The Holocaust in the Secondary classroom: confluence of history, fiction and reality in concentration literature

Raquel Rocamora Montenegro*

\section{Resumen}

Resulta evidente la actual proliferación de títulos con el Holocausto como telón de fondo; basta con echar un vistazo a las estanterías de las librerías, las cuales acogen multitud de volúmenes que encierran en sus páginas este triste capítulo de la historia. El acercamiento a dicho tema se ha planteado desde distintos enfoques desde el siglo XX -literario, histórico e historiográfico, psicológico, filosófico, etc.-, de ahí que haya sido estudiado en la escuela a partir de multitud de propuestas en materias como Educación Ético-Cívica, Geografía e Historia, Lengua Castellana y Literatura, etc. A raíz de la relación entre historia, ficción y realidad, tan en boga en la actualidad debido al éxito de obras basadas en historias reales, surge un debate que ocasiona una reflexión sobre la imagen que ofrecen estos libros. Este puede ser llevado al aula para discurrir acerca de los límites entre la historia y la literatura, a la vez que se fomentan valores y se superan prejuicios con el fin de que no se repita un episodio similar.

Palabras clave: Shoá. Pedagogía del Holocausto. Literatura concentracionaria. Ficción literaria. Memorialismo. Narración testimonial.

\begin{abstract}
The current proliferation of titles with the Holocaust as a backdrop is evident; it can be appreciated just by taking a look at the bookshelves, which house a multitude of volumes that enclose this sad chapter in history in their pages. The approach to this topic has been proposed from different approaches since the 20th century -literary, historical and historiographic, psychological, philosophical, etc.-, hence it has been studied in school from a multitude of proposals in subjects such as Ethical Education, Geography and History, Spanish Language and Literature, etc. As a result of the relationship between history, fiction and reality, so in vogue today due to the success of works based on real stories, a debate arises that causes a reflection on the image offered by these books. This can be taken to the classroom to discuss the limits between history and literature, while promoting values and overcoming prejudices so that a similar episode is not repeated.
\end{abstract}

Key words: Shoah. Holocaust pedagogy. Concentration literature. Literary fiction. Memorialism. Testimonial narration.

\footnotetext{
*Universidad de Alicante•ORCID 0000-0002-4466-8024• raquel.rocamora@ua.es
}

Rocamora, R. (2020). El Holocausto en el aula de Secundaria: confluencia de historia, ficción y realidad en la literatura concentracionaria. CLIO. History and History teaching, 46, 136-147. https://10.26754/ojs clio/clio-2020465282. Recibido: 12/9/2020. Aceptado: 4/12/2020. 


\section{El Holocausto: tema ineludible en el currículum}

Centrar la atención en el Holocausto como fenómeno nunca resulta una tarea sencilla, ya sea por los amargos sentimientos que afloran en dicho acercamiento ante una situación de tal calibre en la sociedad moderna, por la falta de respuestas sencillas ante las múltiples preguntas que surgen o por la cantidad de víctimas inocentes recogidas en lo que puede parecer un simple término. No en vano Brawer (2010) afirma que "el Holocausto (Shoá) es uno de los episodios traumáticos más extremos de la historia de la humanidad. Sus profundas huellas siguen presentes en la cultura universal” (p. 11).

De esto se deduce, precisamente, la necesidad de cumplir con un imperativo de recuerdo asociado a crímenes contra la humanidad perpetrados en nombre del nazismo, pues sus terribles actos se han convertido en un legado compartido del que la humanidad obtiene lecciones universales. En este punto, es necesario recordar que, junto a las víctimas judías, hubo otros grupos duramente perseguidos por el régimen nazi y por sus colaboradores: gitanos, eslavos, prisioneros de guerra soviéticos, comunistas, testigos de Jehová, discapacitados, homosexuales y disidentes políticos y religiosos, entre otros que sufrieron las consecuencias de este cruel sistema.

Tal y como se recoge en una de las paredes de Auschwitz, "los que no recuerdan el pasado están condenados a repetirlo" (Santayana, 1958, p. 16), y nosotros, como docentes e investigadores, y como seres humanos dispuestos a superar antiguos prejuicios y a fomentar valores que incidan en la convivencia pacífica, la empatía y la solidaridad, estamos llamados a dedicar parte de nuestro tiempo a mantener viva la memoria de todos aquellos que se vieron envueltos en uno de los acontecimientos más tristes de la historia ${ }^{1}$ :

Quienes quieren que olvidemos, de alguna manera están del lado de los
victimarios. Recordar a las víctimas exige conocer la verdad. Nosotros tenemos el
deber de transmitir estos acontecimientos a los jóvenes en particular. No somos
tan ingenuos para pensar que solamente con la transmisión estaremos protegidos
contra el regreso de la barbarie. Necesitamos educar, reconstruir los valores, la fe,

\footnotetext{
${ }^{1}$ De la necesidad de educar al alumnado por medio del tratamiento de este tema en el aula se ha hecho eco la prensa (Emergui, 2019; Lendoiro, 2018 y Rodríguez, 2018, entre otros). En consecuencia, existen numerosos recursos educativos que pueden ser consultados en línea ("El Holocausto: un sitio de aprendizaje para estudiantes", del United States Holocaust Memorial Museum; "Materiales educativos", del Yad Vashem, etc.).
} 
la esperanza paciente, terca, obstinada, de que el mundo de mañana será mejor que el de hoy. (Zylberman, 2010, p. 94)

Este acercamiento se ha afrontado, desde el siglo XX, desde numerosos enfoques literario, histórico e historiográfico, psicológico, filosófico, etc.-, los cuales se complementan y enriquecen. De este modo, se han estudiado, entre otras cuestiones, las causas y consecuencias del ascenso del partido nazi en su contexto sociopolítico, las condiciones de vida de los prisioneros en los campos de concentración y las secuelas psicológicas, afectivas y emocionales que marcaron irremediablemente a las personas que lograron sobrevivir a esta barbarie.

Los variados puntos de vista mencionados repercuten, a su vez, en las amplias posibilidades pedagógicas que ofrece el tratamiento de dicho asunto en el aula, ya que son muchas las materias en las que tiene cabida un acercamiento más o menos pormenorizado -Educación Ético-Cívica, Historia y Cultura de las Religiones, Geografía e Historia, Lengua Castellana y Literatura o Historia de la Filosofía, entre otras $^{2}-$, si bien se aconseja trabajar el Holocausto en el aula como actividad transversal en la que cooperen distintas asignaturas como las ya mencionadas.

\section{El punto de vista literario}

Entre los enfoques anteriormente citados, se encuentra el literario, pues son numerosos los documentos de este tipo que acogen como tema central la Shoá o que están, de un modo u otro, vinculados con dicho acontecimiento.

Si bien es cierto que se puede hablar de un silencio en los años inmediatamente posteriores al fin de la Segunda Guerra Mundial en lo que a literatura y testimonios sobre el Holocausto se refiere, desde hace unas décadas, estos relatos han experimentado un notable auge.

A mediados del siglo $X X$, la necesidad o voluntad de olvidar los recientes acontecimientos de la guerra y los terribles campos de concentración, que se

\footnotetext{
${ }^{2}$ Actualmente, son muchas las posibilidades didácticas que se encuentran en Internet a modo de guías docentes con pautas para orientar al profesorado en la enseñanza del Holocausto (Grupo Eleuterio Quintanilla, 2007; Adamoli y Lorenz, 2010; Suárez, M. Á., s. f. y Unidad de Trabajo para la Cooperación Internacional sobre la Enseñanza, Conmemoración e Investigación del Holocausto, s. f., entre otras).
} 
traducían en recuerdos llenos de dolor y sufrimiento, dio paso, de manera progresiva, a la voluntad, de algunos de los supervivientes de la barbarie, de tomar la pluma para dar a conocer al mundo, que había permanecido al margen -ya fuese por desconocimiento o por alianza con el régimen nazi-, los horrores que habían sufrido desde el ascenso al poder del nazismo:

A necessidade de recordar uma experiência extrema impõe-se ao sobrevivente (e antes ainda ao prisioneiro) com uma urgência visceral. Na origem, há o desejo de desabafo, que resulta numa narração irrefletida e convulsiva. Somente o impulso intelectual fornece à memória a tranqüilidade e a serenidade necessárias à comunicação narrativa: é graças à vontade de compreender que a recordação se traduz em relato ${ }^{3}$. (Barenghi, 2005: p. 180)

De esta manera, relataron cómo, en medio del terror que suponía vivir entre alambradas bajo estrictas y temerosas órdenes, malos tratos y vejaciones, conseguían sacar fuerzas para aferrarse a la vida. Estas fuentes documentales constituyen un rico material bibliográfico para dar cuenta a las nuevas generaciones de lo sucedido en ese triste capítulo de la historia. En palabras de Munté (2014, p. 89): "los testimonios son fundamentales y se les reconoce una función pedagógica substancial en la transmisión de su experiencia a las nuevas generaciones. La literatura tiene un papel clave en la difusión de la memoria, con una especial voluntad de transmisión transgeneracional".

No obstante, estas personas no son las únicas que se lanzaron a la tarea de escribir sobre este dantesco acontecimiento, ya que, desde el siglo pasado, distintos intelectuales, entre ellos historiadores, filósofos, filólogos y sociólogos, han sentido la necesidad de acercarse a este tema y de relatar lo sucedido.

En la actualidad, la proliferación de títulos vinculados con el Holocausto es evidente, pues son numerosos los volúmenes recientes que forman parte de las estanterías de las librerías.

Entre estas publicaciones, han visto la luz numerosos títulos que encabezan la portada de obras basadas en supuestas historias reales, los cuales han ocasionado un debate

\footnotetext{
3 "La necesidad de recordar una experiencia extrema se impone al superviviente (e incluso antes al prisionero) con urgencia visceral. Al principio, hay un deseo de desahogarse, lo que resulta en una narración irreflexiva y convulsa. Solo el impulso intelectual proporciona a la memoria la tranquilidad y la serenidad necesarias para la comunicación narrativa: es gracias a la voluntad de comprender que la memoria se traduce en un relato".
} 
acerca de la manera de narrar los horrores de los campos de concentración, pues, en ocasiones, se ha recurrido a múltiples estrategias de ficcionalización y estetización que han hecho un flaco favor a la reivindicación de esta parte del pasado (Conejo, 2016).

De esta manera, se han alzado voces que han defendido la inadecuación de ciertos libros para dar a conocer dicho suceso, precisamente por la imagen superficial, banal y adulterada de lo que realmente ocurrió en los campos de concentración en los que se ambientan los relatos. Entre los detractores de esta literatura se encuentra WitekMalicka (2018), trabajadora del Memorial de Auschwitz, la cual achacó a El tatuador de Auschwitz (2018), de Heather Morris, una falta de rigor histórico fundamentada en el distorsionado reflejo de la vida en dicho campo:

\begin{abstract}
Although the story is built around the fate of an authentic KL Auschwitz prisoner whose stay in the camp and a part of his camp life may be confirmed by surviving archival documentation, the book, however, contains numerous errors and information inconsistent with the facts; as well as overinterpretations, misinterpretations and understatements on which the overall inauthentic picture of the camp reality is built ${ }^{4}$. (p. 6)
\end{abstract}

Esto mismo ocurrió en su momento con la afamada obra de John Boyne El niño con el pijama de rayas (2006), pues el Memorial (2020a, tuit) desaconsejó su lectura para aquellos que deseasen conocer la verdadera historia de la Shoá -“[...] 'The Boy in the Striped Pyjamas' should be avoided by anyone who studies or teaches factual history of the \#Holocaust'5-. En cambio, Piotr Setkiewicz (2020b, tuit), director del Centro de Investigación del Memorial de Auschwitz, citó como títulos adecuados algunos testimonios de los supervivientes de dicha barbarie:

Books recommended by the head of our Research Center Dr. Piotr Setkiewicz: Halina Birenbaum - "Hope is the last to Die", Tadeusz Borowski - "This Way for the Gas, Ladies and Gentlemen", Charlotte Delbo - "Auschwitz and After", Viktor E. Frankl - "Man's Search for Meaning", Imre Kertész - "Fatelessness", Rena Kornreich Gelissen - "Rena's Promise: A Story of Sisters in Auschwitz", Primo Levi - "If This Is a Man" (Survival in Auschwitz) / The Truce (The Reawakening), Witold Pilecki -

\footnotetext{
4 "Aunque la historia se basa en el destino de un auténtico prisionero de KL Auschwitz, cuya estancia en el campo y una parte de su vida en él puede ser confirmada por la documentación de archivo de sobrevivientes, el libro, sin embargo, contiene numerosos errores e información incongruente respecto a los hechos, así como sobreinterpretaciones, malas interpretaciones y subestimaciones sobre las que se construye la imagen inauténtica general de la realidad del campo".

5 “[...] 'El niño con el pijama de rayas' debe ser evitado por cualquier persona que estudie o enseñe la historia fáctica del \#Holocausto".
} 
"Witold's Report from Auschwitz", Seweryna Szmaglewska - "Smoke over Birkenau", Elie Wiesel - "Night" \& "Amidst a Nightmare of Crime: Manuscripts of Prisoners in Crematorium Squads Found at Auschwitz (The Scrolls of Auschwitz)" .

Lo que todos comparten es, precisamente, el hecho de ser relatos narrados por aquellos que se vieron obligados a vivir en las más horrendas circunstancias que puede experimentar el ser humano al encontrarse en un lugar remoto despojado de su identidad y dignidad, de ahí que constituyan fuentes primarias en tanto en cuanto proceden del puño y letra de sus protagonistas y no de escritores, investigadores y, en definitiva, personas ajenas que estudian posteriormente lo sucedido.

\section{Encrucijada entre literatura e historia}

El origen del debate se sitúa en la confluencia o relación entre historia, ficción y realidad, pues, siguiendo a Jitrik (1995), "la ficción, como idea, atañe a la novela y aparece en escena casi al mismo tiempo que el historicismo; no es de extrañar que entre ambos términos se haya establecido una conexión" (pp. 10-11). Por lo tanto, en el caso de aquellos volúmenes redactados por los supervivientes ${ }^{7}$, cabría hablar de una vinculación entre ambas disciplinas -literatura e historia- más que de una disyuntiva, pues, en ocasiones, resulta compleja la tarea de tratar de deslindar los límites entre la realidad, vinculada con la historia, y la ficción, plano al que pertenecen ciertas distorsiones que pueden hallarse en la narración de los supervivientes, ya que los sucesos pasan, necesariamente, por el filtro de su subjetividad para relatar de un modo u otro los horrores de acuerdo a su propia experiencia:

\footnotetext{
${ }^{6}$ Libros recomendados por el director de nuestro Centro de Investigación, el Dr. Piotr Setkiewicz: Halina Birenbaum - "La esperanza es la última en morir", Tadeusz Borowski - "This Way for the Gas, Ladies and Gentlemen", Charlotte Delbo - "Auschwitz y después", Viktor E. Frankl - "El hombre en busca de sentido", Imre Kertész - "Sin destino", Rena Kornreich Gelissen - "Rena's Promise: A Story of Sisters in Auschwitz", Primo Levi - "Si esto es un hombre" (Supervivencia en Auschwitz) / La tregua (El despertar)", Witold Pilecki - "Witold's Report from Auschwitz", Seweryna Szmaglewska - "Una mujer en Birkenau” [sic], Elie Wiesel - "La noche" y "Amidst a Nightmare of Crime: Manuscripts of Prisoners in Crematorium Squads Found at Auschwitz (The Scrolls of Auschwitz)". Únicamente se han traducido aquellos títulos disponibles también en español y se ha añadido, en todos los casos, la cursiva al tratarse de títulos de obras.

${ }^{7}$ Este es el caso de los citados anteriormente, a los cuales se pueden añadir, entre otros, El último judío de Treblinka: una memoria (1945), de Chil Rajchman; Auschwitz, última parada: Cómo sobreviví al horror (1943-1945) (1946), de Eddy de Wind; El largo viaje (1963), de Jorge Semprún; Ninguno de nosotros volverá (1965), de Charlotte Delbo y Necrópolis (1967), de Boris Pahor, algunos de ellos no escritos por personas judías, sino que pertenecían a otros colectivos que también fueron perseguidos por el régimen nazi y sus colaboradores, como los ya señalados anteriormente -romaníes, homosexuales, prisioneros de guerra, discapacitados, etc.-.
} 
La memoria es todo menos una reproducción fiel del pasado, pues mezcla dos tipos de experiencia, la vivida y la transmitida, a partir de lo cual es difícil saber hasta qué punto la narración proveniente de esa memoria es pura, y más aun cuando estas experiencias son fruto de algo tan atroz como lo sucedido dentro de los campos. (Conejo, 2016, p. 63)

En el momento de tomar la pluma, los exprisioneros escogen qué parte de su historia desean contar, ya sea sin filtros o con algunos detalles omitidos, mientras que, en el caso de aquellas personas ajenas a lo sucedido, el relato distorsionado puede provenir, además, de una laguna documental o de la voluntad de ficcionalizar 0 inventar algunos detalles de su narración como información añadida, independientemente del motivo que subyazca detrás - ganar en dramatismo, adornar escenas con sucesos no acontecidos, añadir personajes con una conducta determinada que no existieron en la realidad, etc.-.

El problema adquiere mayores dimensiones cuando escritores actuales añaden, en sus sobrecubiertas y portadas, el sintagma "historia real", como ocurre en Un amor en Auschwitz (2017), de Francesca Paci, en El chico que siguió a su padre hasta Auschwitz (2018), de Jeremy Dronfield, en El maestro de Auschwitz (2020), de Antonio Iturbe y en el ya citado El tatuador de Auschwitz, todas ellas historias ajenas sobre las que se ha investigado posteriormente.

Dicha afirmación lleva a pensar, de manera errónea, que la información contenida en estos volúmenes cumple fielmente con los principios de veracidad histórica. Lo cierto es que, para construir la narración resultante, por lo general, se entremezclan testimonios y datos reales con descripciones y detalles pertenecientes a un plano ficcional y accesorio, como se ha ejemplificado anteriormente con las opiniones del Memorial sobre las obras aludidas.

Como consecuencia, el lector no encontrará en estos libros un retrato absolutamente veraz debido a las licencias que se toman los autores al escribirlos, que los alejan de la escritura basada en el rigor histórico. 


\section{El estudio del Holocausto como proyecto transversal}

La reflexión sobre la relación entre historia, ficción y realidad es, precisamente, una de las que se pueden trasladar al aula a raíz del estudio del memorialismo, la novela histórica y la literatura testimonial, de manera que se acerque al alumnado a este triste acontecimiento desde la materia de Lengua Castellana y Literatura.

El planteamiento se enriquecería todavía más si se programase una colaboración con otras asignaturas del currículum, de manera que el tratamiento del tema se abordase desde un punto de vista multidisciplinar lo más completo posible. Para ello, se podría presentar al alumnado un proyecto transversal a propósito del Día Internacional de Conmemoración en Memoria de las Víctimas del Holocausto, celebrado anualmente el 27 de enero.

De esta manera, los textos se enriquecerían con otras fuentes primarias, como materiales audiovisuales, que podrían ser analizados en la asignatura de Geografía e Historia, los cuales se complementarían con reflexiones sobre el sentido de la existencia y de la justicia y los límites del ser humano, pertenecientes a las materias de Historia de la Filosofía y de Educación Ético-Cívica, y con el fomento de valores como la solidaridad y el respeto por diversas creencias en la asignatura de Historia y Cultura de las Religiones.

Con todo esto, se estaría contribuyendo al desarrollo integral del alumnado mediante la elaboración de un juicio crítico propio para extraer conclusiones sobre el pasado que les sirvan para afrontar su presente y, sobre todo, su futuro; un objetivo fundamental en los centros de enseñanza. En palabras de Baer, "la manera en que recordamos y representamos el pasado significa también cómo nos situamos en el presente, cómo miramos hacia el futuro" (comunicación personal, 30 de septiembre de 2020), pues condiciona nuestras formas de acción colectiva y la transmisión de deberes y valores a las siguientes generaciones.

Al fin y al cabo, no se debe olvidar que, además de transmitir conocimientos teóricoprácticos sobre las distintas materias que forman parte del currículum escolar, el papel del docente es educar en colaboración con las familias, y una de las maneras idóneas de hacerlo es ofreciendo respeto y rindiendo tributo a aquellos que padecieron dicha 
barbarie. El fin, de acuerdo con el imperativo moral de la ética del "nunca más" (Baer y Sznaider, 2016), no es otro que evitar proyectar en el futuro grandes e irrevocables errores del pasado de los que es necesario aprender.

\section{El Holocausto en Lengua Castellana y Literatura: Un saco de canicas (1973), de Joseph Joffo y El arca de Schindler (1982), de Thomas Keneally}

Desde el área de Lengua Castellana y Literatura, en el nivel de Secundaria se podrían abordar dos lecturas que se debatirían posteriormente en gran grupo con el objetivo de reflexionar en torno a la literatura concentracionaria, la cual participa de la novela histórica, el memorialismo y la narrativa testimonial, a la vez que se forja la personalidad de los estudiantes y se fomentan valores necesarios tanto en la escuela como en el exterior. Siguiendo, de nuevo, a Baer (comunicación personal, 30 de septiembre de 2020), el sentido de la memoria se traduce en las lecciones que los seres humanos atribuimos al pasado, en este caso al del Holocausto, que implicó el exterminio sistemático de millones de judíos. El mensaje universalista que transmite este suceso está relacionado con principios de tolerancia, inclusión, respeto a la diversidad cultural y defensa de los derechos humanos; valores que se quebraron durante el nazismo.

Este tema se podría abordar en la asignatura de Lengua Castellana y Literatura mediante la reflexión sobre la imagen que ofrecen estos libros a partir de la confluencia de historia, ficción y realidad aludida anteriormente. De este modo, se produciría el acercamiento a los subgéneros anteriormente mencionados, lo cual daría lugar a un debate entre los estudiantes sobre las posibilidades narrativas, a la vez que se fomentan los valores ya mencionados.

En el momento de decidir los títulos para cada etapa, se habría de tener presente el nivel emocional y la madurez del alumnado, pues no todos ellos reflejan con la misma crudeza y prolijidad los horrores que padecieron los prisioneros. Sin embargo, en todos los casos, se trataría de lecturas que contienen grandes lecciones de humanidad, de amor por el prójimo y de esperanza, ya que sus protagonistas ejemplifican cómo el ser humano luchó por sobrevivir en las condiciones más adversas durante uno de los capítulos más tristes y oscuros de la historia. 
Atendiendo a lo comentado anteriormente, se podría plantear como lectura en este proyecto transversal para los cursos de Secundaria Un saco de canicas (1973), de Joseph Joffo y El niño de Schindler (1982), de Leon Leyson; en ambos casos relatos basados en las historias de los propios autores, de manera que pretenden ser autobiográficos; sin embargo, como se ha apuntado anteriormente, no se debe olvidar que "cada cual cuenta sus experiencias a su manera pasando por el filtro selectivo de aquello que quieren expresar" (Conejo, 2016, p. 66). Pese a ello, se trata de dos obras vinculadas con la literatura histórica y memorialista, en las cuales, siguiendo a Lukács (1977), lo importante es que "se plasmen destinos individuales tales que se expresen en ellos en forma inmediata y a la vez típica los problemas vitales de la época" ( $p$. 354). La obra de Joseph Joffo acoge a personajes cuyos destinos muestran la necesidad de huir para tratar de poner a salvo su vida, mientras que la de Leon Leyson reúne a individuos que luchan por sobrevivir en las condiciones más adversas que se puedan imaginar: el intento de exterminio de todo un pueblo.

En el primer volumen, Joseph Joffo relata cómo se ve obligado a separarse de su familia y a huir junto a su hermano Maurice de la Francia ocupada por los nazis en un intento de salvaguardar su vida hasta que consiga reunirse con su familia de nuevo tras la guerra. Para ello, habrá de superar numerosas adversidades que harán que su infancia termine precipitadamente a la edad de 10 años, en un exitoso intento de sortear los temibles campos de concentración.

En el segundo, Leon Leyson, el menor de los schindlerjuden -judíos de Oskar Schindler-, rememora sus recuerdos y relata sus primeros años de vida, que estuvieron marcados por el traslado al gueto y la posterior marcha al campo de concentración de Auschwitz. Gracias a la intervención de Schindler, un alemán católico que, siendo miembro del partido nazi, sacrificó su fortuna y arriesgó su vida para conseguir salvar la de más de mil judíos que iban a ser exterminados, Leon consiguió sobrevivir.

Estas lecturas se podrían complementar, posteriormente, con la visualización de las adaptaciones cinematográficas Una bolsa de canicas (2017), dirigida por Christian Duguay y La lista de Schindler (1993), dirigida por Steven Spielberg y galardonada en los Premios Óscar. La reproducción de una cinta cinematográfica, además de ser clarificadora, resulta atractiva para el alumnado, ya que permite ver reflejados en 
imágenes los sucesos leídos con anterioridad, que a tantas interesantes reflexiones habrán dado lugar. Además, su visualización posibilitará traducir las cifras y los datos teóricos en personas reales, de manera que podrá producirse un mayor acercamiento por parte de los estudiantes al tema en cuestión en un intento de compartir el dolor de quienes sufrieron tales atrocidades y de aprender de ello.

\section{Referencias}

Adamoli, M. C. y Lorenz, F. (Eds.) (2010). Holocausto: Preguntas, respuestas y propuestas para su enseñanza. Ministerio de Educación de la Nación Argentina. http://www.bnm.me.gov.ar/giga1/documentos/EL005100.pdf

Auschwitz Memorial. [@AuschwitzMuseum]. (2020a). [...] "The Boy in the Striped Pyjamas" should be avoided by anyone who studies or teaches factual history of the \#Holocaust [tuit]. Twitter. https://twitter.com/AuschwitzMuseum/status/1213823424969224193

Auschwitz Memorial. [@AuschwitzMuseum]. (2020b). Books recommended by the head of our Research Center Dr. Piotr Setkiewicz [tuit]. Twitter. https://twitter.com/AuschwitzMuseum/status/1213825253656453123

Baer, A. y Sznaider, N. (2016). Memory and Forgetting in the Post-Holocaust Era: The Ethics of Never Again. Routledge.

Baer, A. (2020, 30 de septiembre). La memoria y el olvido en la era post-Holocausto: La ética del "Nunca más" [sesión de conferencia]. Centro Sefarad-Israel, Madrid, España. https://www.ivoox.com/memoria-olvido-era-audios-mp3 if 57297573 1.html

Barenghi, M. (2005). A memória da ofensa: recordar, narrar, compreender (M. Santana, trad.). Novos estudos, 73, 175-191 (original publicado en 2000). https://www.scielo.br/pdf/nec/n73/a13n73.pdf

Brawer, M. (2010). Un compromiso activo con los derechos humanos. En M. C. Adamoli, A. Roisenstraj y G. Jinich (Comps.), La enseñanza del Holocausto/Shoá como acontecimiento clave del siglo XX. Aportes para una agenda educativa en tiempo presente (pp. 11-12). Ministerio de Educación, Subsecretaría de Equidad y Calidad Educativa y Museo del Holocausto de Buenos Aires.

Conejo, Y. (2016). Los límites de la representación y la reflexión en torno al problema de la ficcionalización y estetización en la literatura de posguerra, el caso de los sobrevivientes. $\begin{array}{llll}\text { Memoria } y & \text { sociedad, 20(40), 58-69. }\end{array}$ https://dialnet.unirioja.es/servlet/articulo?codigo $=5513157$

Emergui, S. (2019, 3 de septiembre). Aprender a enseñar el Holocausto. El Mundo. https://www.elmundo.es/internacional/2019/09/03/5d6e633f21efa0ef558b45d4.html

Grupo Eleuterio Quintanilla. (2007). Pensad que esto ha sucedido. Guía de recursos para el estudio del holocausto. Tercera Prensa-Gakoa.

Jitrik, N. (1995). Historia e imaginación literaria: las posibilidades de un género. Biblos. 
Lendoiro, G. (2018, 25 de enero). La importancia de enseñar en las escuelas qué fue el Holocausto, recordar para no olvidar. La Razón. https://www.lavanguardia.com/vida/20180630/45502254077/aprendiendo-a-ensenar-elholocausto.html

Lukács, G. (1977). La novela histórica (J. Reuter, trad.). Era.

Munté, R. À. (2014). Reseña de Arráez, J. L. y Peral, A. (Eds.) (2012). Memoria de la Shoá: Literatura y testimonio, Sefarad editores. Anuari de filologia. Literatures contemporànies, 4, 89-93. https://dialnet.unirioja.es/servlet/articulo?codigo $=6000879$

Rodríguez, P. (2018, 30 de junio). Aprendiendo a enseñar el Holocausto. La Vanguardia. https://www.lavanguardia.com/vida/20180630/45502254077/aprendiendo-a-ensenar-elholocausto.html

Santayana, J. (1958). La vida de la razón. (R. M. Agoglia, trad.). Nova.

Suárez, M. Á. (s. f.). La enseñanza del Holocausto en Bachillerato: Reflexiones y Propuestas. Yad Vashem Centro Mundial de Conmemoración de la Shoá. https://www.yadvashem.org/es/education/educational-materials/proposals/reflections.html

Unidad de Trabajo para la Cooperación Internacional sobre la Enseñanza, Conmemoración e Investigación del Holocausto. (s. f.). Manual para docentes. Guía para la enseñanza del Holocausto.http://holocaustmusic.ort.org/fileadmin/user upload/ imported/fileadmin/down loads/guia para ensenanza del holocausto.pdf

United States Holocaust Memorial Museum. (s. f.). El Holocausto: un sitio de aprendizaje para estudiantes. https://encyclopedia.ushmm.org/content/es/project/the-holocaust-a-learningsite-for-students

Witek-Malicka, W. (2018). Fact-checking 'The Tattooist of Auscwhtiz'. Memoria magazine, 14, 16-17. https://view.joomag.com/memoria-en-no-14-11-2018/0766192001543510530

Yad Vashem (s. f.). Materiales educativos. https://www.yadvashem.org/ es/education/educational-materials.html

Zylberman, A. (2010). Ideas y prácticas genocidas en el nacionalsocialismo: el caso del pueblo judío. En M. C. Adamoli, A. Roisenstraj y G. Jinich (Comps.), La enseñanza del Holocausto/Shoá como acontecimiento clave del siglo XX. Aportes para una agenda educativa en tiempo presente (pp. 85-94). Ministerio de Educación, Subsecretaría de Equidad y Calidad Educativa y Museo del Holocausto de Buenos Aires. 